\title{
Constraints on the Timeon Model
}

\author{
Takeshi Araki and C. Q. Geng \\ Department of Physics, National Tsing Hua University, Hsinchu, Taiwan 300.
}

\begin{abstract}
The timeon model recently proposed by Friedberg and Lee has a potential problem of flavor changing neutral currents (FCNCs) if the mass of the timeon is small. In order to avoid, we introduce a small dimensionless parameter to suppress FCNCs. Even in this case, we find that the timeon mass must be larger than $151 \mathrm{GeV}$ to satisfy all the constraints from processes involving FCNCs in the quark sectors. We also extend the timeon model to the lepton sector and examine the leptonic processes.
\end{abstract}




\section{INTRODUCTION}

$C P$ violation $(\mathrm{CPV})$ is one of the most important features in particle physics. In the standard model (SM), it arises from a unique physical phase of the Cabibbo-KobayashiMaskawa (CKM) quark mixing matrix [1]. However, the origin of CPV remains unclear. On the other hand, it is unknown whether CPV also occurs in the lepton sector.

In general, there are two possible sources of CPV: complex couplings of the Yukawa interactions and complex vacuum expectation values (VEVs) of the scalar fields, which explicitly and spontaneously break $C P$ symmetry, such as the cases of the SM and multiHiggs doublet models [2], respectively.

Recently, a new spontaneous $C P$ violation mechanism has been proposed by Friedberg and Lee [3]. They introduced a gauge singlet pseudoscalar which couples to quarks to yield a new type of Yukawa interactions. As the Yukawa couplings are assumed to be real, the system is $C P$ and $T$ conserving. Once the pseudoscalar acquires a VEV, both $C P$ and $T$ are spontaneously broken. The excitation of the pseudoscalar from its vacuum predicts a new neutral scalar field named as "timeon". One of the most interesting features of the proposal is that the pseudoscalar is also responsible for the masses of up and down quarks, but not for the heavy quarks. As a result, the timeon mass and the VEV can be much lower than electroweak scale.

The timeon model, however, has a potential problem of flavor changing neutral currents (FCNCs) mediated by the timeon. As we shall explain later, the timeon mass is strongly constrained by the mass mixings of the neutral mesons and must be much larger than its VEV. This fact implies the strongly interacting quartic timeon coupling and the broken down of the perturbation. In this paper, in order to save the model, we introduce a small parameter $\epsilon$ and re-define the VEV as $\tau_{0} \rightarrow \epsilon \tau_{0}$. We will demonstrate that the small $\epsilon$ can relax the phenomenological constraints, which permit a much smaller mass of the timeon. We would also extend the timeon model to the lepton sector and explore leptonic FCNC processes, such as the lepton number violating decays and the muon anomalous magnetic moment.

The paper is organized as follows. In Sec. 2, we give a brief review of the timeon model and calculate the mixing parameters in the neutral meson systems. In Sec. 3, we extend the timeon model to the lepton sector. We present the summary in Sec. 4. 


\section{QUARK SECTOR}

\section{A. Model}

In the theory of timeon, proposed by Friedberg and Lee [3] , the quark mass Hamiltonian is written as

$$
\begin{aligned}
\mathcal{H}_{q} & =\bar{u}_{i}\left[G_{u}+i \gamma_{5} \tau_{0} F\right]_{i j} u_{j}+\bar{d}_{i}\left[G_{d}+i \gamma_{5} \tau_{0} F\right]_{i j} d_{j} \\
& =\bar{u}_{L i}\left[G_{u}+i \tau_{0} F\right]_{i j} u_{R j}+\bar{d}_{L i}\left[G_{d}+i \tau_{0} F\right]_{i j} d_{R j}+\text { h.c. }
\end{aligned}
$$

where the constant $\tau_{0}$ is the VEV of the new $C P$ odd and T odd, gauge singlet pseudoscalar field $\tau(x), i, j=1, \cdots, 3$ stand for family indices and $G_{u, d}$ and $F$ are $3 \times 3$ matrices, given by

$$
G_{u, d}=\left(\begin{array}{ccc}
b_{u, d} \eta_{u, d}^{2}\left(1+\xi_{u, d}^{2}\right) & -b_{u, d} \eta_{u, d} & -b_{u, d} \xi_{u, d} \eta_{u, d} \\
-b_{u, d} \eta_{u, d} & b_{u, d}+a_{u, d} \xi_{u, d}^{2} & -a_{u, d} \xi_{u, d} \\
-b_{u, d} \xi_{u, d} \eta_{u, d} & -a_{u, d} \xi_{u, d} & a_{u, d}+b_{u, d}
\end{array}\right)
$$

and

$$
F=\left(\begin{array}{ccc}
\cos ^{2} \alpha_{q} & \sin \alpha_{q} \cos \alpha_{q} \cos \beta_{q} & \sin \alpha_{q} \cos \alpha_{q} \sin \beta_{q} \\
\sin \alpha_{q} \cos \alpha_{q} \cos \beta_{q} & \sin ^{2} \alpha_{q} \cos ^{2} \beta_{q} & \sin ^{2} \alpha_{q} \sin \beta_{q} \cos \beta_{q} \\
\sin \alpha_{q} \cos \alpha_{q} \sin \beta_{q} & \sin ^{2} \alpha_{q} \sin \beta_{q} \cos \beta_{q} & \sin ^{2} \alpha_{q} \sin ^{2} \beta_{q}
\end{array}\right)
$$

with the real parameters of $a_{u, d}, b_{u, d}, \eta_{u, d}$ and $\xi_{u, d}$ and two angles of $\alpha_{q}$ and $\beta_{q}$, respectively. Furthermore, $\eta_{u, d}$ and $\xi_{u, d}$ in Eq. (2) can be also defined by four angles as

$$
\begin{aligned}
& \xi_{u}=\tan \phi_{u}, \eta_{u}=\tan \varphi_{u} \cos \phi_{u}, \\
& \xi_{d}=\tan \phi_{d}, \eta_{d}=-\tan \varphi_{d} \cos \phi_{d} .
\end{aligned}
$$

The potential of the pseudoscalar field is given by [3]

$$
V(\tau)=-\frac{1}{2} \lambda \tau^{2}\left(\tau_{0}^{2}-\frac{1}{2} \tau^{2}\right)
$$

which leads to the mass of the new quantum, i.e., timeon, to be

$$
M_{T}=(2 \lambda)^{\frac{1}{2}} \tau_{0}
$$


As seen from Eq. (2), $G_{u, d}^{*}=G_{u, d}$ and $\operatorname{det} G_{u, d}=0$, and thus up and down quarks of the first generation are massless before $\tau(x)$ receives the VEV. After $\tau(x)$ gets the VEV, $C P$ and $T$ are spontaneously broken and the first generation quarks acquire small masses. Note that since the timeon is not responsible for the masses of heavy quarks, its VEV can be much lower than the electroweak scale.

\section{B. Flavor Changing Neutral Currents}

In Ref. [3], it was estimated that $\tau_{0} \simeq 33 \mathrm{MeV}$, which implies that

$$
M_{T} \leq 47 \mathrm{MeV}
$$

from Eq. (7) if $\lambda \leq 1$. Therefore, the mass of the timeon is much lower than the electroweak scale. However, a light timeon could give rise to dangerous FCNC processes because in general $G_{u(d)}$ and $F$ cannot be diagonalized simultaneously. In fact, in the mass eigenstate basis of quarks, flavor changing timeon couplings are given by

$$
\begin{gathered}
F^{u} \equiv U_{u}^{T} F U_{u}=\left(\begin{array}{ccc}
0.08 & -0.14 & 0.23 \\
-0.14 & 0.25 & -0.41 \\
0.23 & -0.41 & 0.67
\end{array}\right), \\
F^{d} \equiv U_{d}^{T} F U_{d}=\left(\begin{array}{ccc}
0.15 & -0.18 & 0.31 \\
-0.18 & 0.21 & -0.36 \\
0.31 & -0.36 & 0.64
\end{array}\right),
\end{gathered}
$$

where $U_{u}$ and $U_{d}$ are the unitary matrices to diagonalize up and down-type quarks, respectively. From the values in Eq. (9) and inputs in Table I1, the mass mixing parameter in the neutral $K$ meson system due to the timeon contribution at tree level is estimated to be

$$
\begin{aligned}
& \Delta M_{K}^{\text {timeon }}=2\left|\frac{2 F_{d s}^{d}\left(F_{s d}^{d}\right)^{*}}{M_{T}^{2}}<K^{0}\right| \bar{d}_{L}^{\alpha} s_{R}^{\alpha} \bar{d}_{R}^{\beta} s_{L}^{\beta} \mid \bar{K}^{0}> \\
& \quad+\frac{\left(F_{d s}^{d}\right)^{2}+\left(F_{s d}^{d}\right)^{* 2}}{M_{T}^{2}}<K^{0}\left|\bar{d}_{L}^{\alpha} s_{R}^{\alpha} \bar{d}_{L}^{\beta} s_{R}^{\beta}\right| \bar{K}^{0}>\mid \\
& \\
& \sim \frac{1.76 \times 10^{-3} \mathrm{GeV}^{3}}{M_{T}^{2}} .
\end{aligned}
$$




\begin{tabular}{|c|c||c|c|}
\hline parameter & input & parameter & input \\
\hline$m_{u}(2 \mathrm{GeV})$ & $2.5 \times 10^{-3} \mathrm{GeV}$ & $f_{K}$ & $0.16 \mathrm{GeV}$ \\
\hline$m_{d}(2 \mathrm{GeV})$ & $5 \times 10^{-3} \mathrm{GeV}$ & $f_{B_{s}}$ & $0.24 \mathrm{GeV}$ \\
\hline$m_{s}(2 \mathrm{GeV})$ & $0.095 \mathrm{GeV}$ & $f_{B_{d}}$ & $0.198 \mathrm{GeV}$ \\
\hline$m_{c}\left(m_{c}\right)$ & $1.25 \mathrm{GeV}$ & $f_{D}$ & $0.223 \mathrm{GeV}$ \\
\hline$m_{b}\left(m_{b}\right)$ & $4.2 \mathrm{GeV}$ & $M_{K}$ & $0.497 \mathrm{GeV}$ \\
\hline$M_{B_{s}}$ & $5.366 \mathrm{GeV}$ & $M_{B_{d}}$ & $5.280 \mathrm{GeV}$ \\
\hline$M_{D}$ & $1.865 \mathrm{GeV}$ & & \\
\hline
\end{tabular}

TABLE I: Values of parameters used in the calculation. Here, we have used the same quark masses as those in Ref. [3], while the decay constants and the meson masses are the center values in Ref. [4].

From the experimental data of $\Delta M_{K}^{e x p} \simeq 3.5 \times 10^{-15} \mathrm{GeV}$, without loss of generality we find the lower limit of the timeon mass as

$$
M_{T} \geq 7 \times 10^{5} \mathrm{GeV}
$$

which is much higher than that in Eq. (8). Here, we have ignored the renormalization group effects as well as some hadronic uncertainties. We note that the mixing parameters in other neutral meson systems also lead to similar constraints on $M_{T}$. Therefore, in the timeon model of Ref. [3], $M_{T} \gg \tau_{0}$, which requires the strongly interacting quartic timeon coupling, i.e., $\lambda \gg 1$.

In order to solve the problem, in this paper, we introduce a small dimensionless parameter $\epsilon$ for the terms related to $F$ in Eq. (11), which is equivalent to a re-defined VEV of

$$
\tau_{0} \rightarrow \epsilon \tau_{0} \simeq 33 \mathrm{MeV}
$$

If $\epsilon$ is very small, the constraints from the FCNC processes can be relaxed becouse the left hand side of Eq. (11) is replaced by $M_{T} / \epsilon$. In this case, the constraint from $\Delta M_{K}$ becomes

$$
M_{T} \geq 151 \mathrm{GeV}
$$


By assuming that $\tau_{0} \simeq M_{T} \sim 151 \mathrm{GeV}$, i.e., $\lambda \simeq 1 / 2$, we obtain that $\epsilon \sim 0.22 \times 10^{-3}$ from Eq. (12). However, If we use a smaller $\tau_{0}$ or a larger $\epsilon, M_{T}$ has to be larger than $151 \mathrm{GeV}$. In this paper, we take $151 \mathrm{GeV}$ as the lowest value of the timeon mass. Consequently, we can also estimate the mixings in $B_{s}, B_{d}$ and $D$ meson systems from the timeon contributions. By using $M_{T} \sim 151 \mathrm{GeV}$, we obtain

$$
\begin{aligned}
& \Delta M_{B_{s}}^{\text {timeon }} \sim 0.365 \times 10^{-13} \mathrm{GeV}<\Delta M_{B_{s}}^{\text {exp }} \simeq 1.17 \times 10^{-11} \mathrm{GeV} \\
& \Delta M_{B_{d}}^{\text {timeon }} \sim 0.178 \times 10^{-13} \mathrm{GeV}<\Delta M_{B_{d}}^{\text {exp }} \simeq 3.34 \times 10^{-13} \mathrm{GeV} \\
& \Delta M_{D}^{\text {timeon }} \sim 0.205 \times 10^{-14} \mathrm{GeV}<\Delta M_{D}^{\text {exp }} \simeq 1.4 \times 10^{-14} \mathrm{GeV} .
\end{aligned}
$$

It is clear that all the FCNC processes are suppressed if $\epsilon \leq 0.22 \times 10^{-3}$ and $M_{T} \geq$ $151 \mathrm{GeV}$.

Now we would like to briefly comment on the possible origin of the small parameter $\epsilon$. Since $\tau(x)$ is a gauge singlet scalar field, it cannot be incorporated into the renormalizable $S U(2)_{L} \times U(1)_{Y}$ model. One way out is to consider a non-renormalizable interacting term, such as,

$$
\frac{F_{i j}}{\Lambda} \bar{\psi}_{L i} \Phi \psi_{R j} \tau
$$

where $\Phi$ is a $S U(2)_{L}$ doublet scalar and $\Lambda$ is a typical energy scale of the model. In this case, $\langle\Phi>/ \Lambda$ might be able to explain the existence of the small parameter $\epsilon$. As the construction of realistic models goes beyond our purpose of this paper, we do not discuss this point further.

\section{LEPTON SECTOR}

\section{A. Model}

In this section, we extend the timeon model to the lepton sector. The lepton mass Hamiltonian is similar to Eq. (1), given by

$$
\mathcal{H}_{l}=\bar{\ell}_{i}\left[G_{\ell}+i \gamma_{5} \epsilon \tau_{0} F\right]_{i j} \ell_{j}+\bar{\nu}_{i}^{(c)}\left[G_{\nu}+i \gamma_{5} \epsilon_{\nu} \tau_{0} F\right]_{i j} \nu_{j}
$$

where $\ell_{i}$ and $\nu_{i}$ represent charged leptons and neutrinos, respectively. Note that in the charged lepton sector, we use the same parameter $\epsilon$ as that of the quark sector, whereas 
in the neutrino sector we assume a different small parameter $\epsilon_{\nu}$ as neutrino masses would be due to some new physics such as the See-Saw mechanism, which may have a different origin from that of the charged fermions ${ }^{1}$. The matrix $F$ takes the same form as Eq. (3) but described by different angles $\alpha_{l}$ and $\beta_{l}$. For $G_{\ell, \nu}$, we assume the following simple textures of

$$
\begin{gathered}
G_{\ell}=\left(\begin{array}{ccc}
0 & 0 & 0 \\
0 & b_{\ell}+a_{\ell} & a_{\ell} \\
0 & a_{\ell} & a_{\ell}+b_{\ell}
\end{array}\right), \\
G_{\nu}=\left(\begin{array}{ccc}
\frac{1}{2} b_{\nu} & \sqrt{\frac{1}{2}} b_{\nu} & 0 \\
\sqrt{\frac{1}{2}} b_{\nu} & b_{\nu} & 0 \\
0 & 0 & a_{\nu}+b_{\nu}
\end{array}\right),
\end{gathered}
$$

respectively. Note that this corresponds to $\eta_{\ell}=\xi_{\nu}=0, \xi_{\ell}=-1$ and $\eta_{\nu}=-\sqrt{\frac{1}{2}}[5]$.

Here, we would like to comment on several features of the model. As shown in Ref. [5], Eqs. (19) and (20) imply the exact tri-bimaximal lepton mixing matrix [6]. That is,

$$
\sin ^{2} \theta_{12}=\frac{1}{3}, \sin ^{2} \theta_{23}=\frac{1}{2}, \sin ^{2} \theta_{13}=0 .
$$

After $\tau(x)$ receives the $\mathrm{VEV}$, the mixing matrix deviates from exact tri-bimaximal mixing. However, if $\alpha_{l}=0$, there is no flavor changing timeon coupling in the charged lepton sector, while $\sin ^{2} \theta_{13}=0$ and $\sin ^{2} \theta_{23}=1 / 2$ remain. On the other hand, if $\alpha_{l} \neq 0, \sin ^{2} \theta_{13}$ and $\sin ^{2} \theta_{23}$ will be different from 0 and $1 / 2$, respectively, and flavor changing timeon couplings in the charged lepton sector are induced. As in the case of the quark sector, $C P$ is spontaneously broken. We note that if $\alpha_{l}=0$, the $C P$ violating Dirac phase in the neutrino sector will not show up due to $\sin ^{2} \theta_{13}=0$.

Since our model is a special case of Ref. [3], the analytic study is the same as that in Ref. [3]. Because the values of $\epsilon$ and $\tau_{0}$ are already determined as $\epsilon=0.22 \times 10^{-3}$ and $\tau_{0}=151 \mathrm{GeV}$ in the quark sector, the model is described by seven parameters: $a_{\ell}, b_{\ell}, a_{\nu}, b_{\nu}, \alpha_{l}, \beta_{l}$ and $\epsilon_{\nu}$, which can be fixed by seven physical quantities. In our numerical calculation, we take [7]

$$
m_{e}=0.511 \mathrm{MeV}, m_{\mu}=105.658 \mathrm{MeV}, m_{\tau}=1776.84 \pm 0.17 \mathrm{MeV}
$$

\footnotetext{
1 We do not restrict ourselves to the case of Majorana neutrinos.
} 


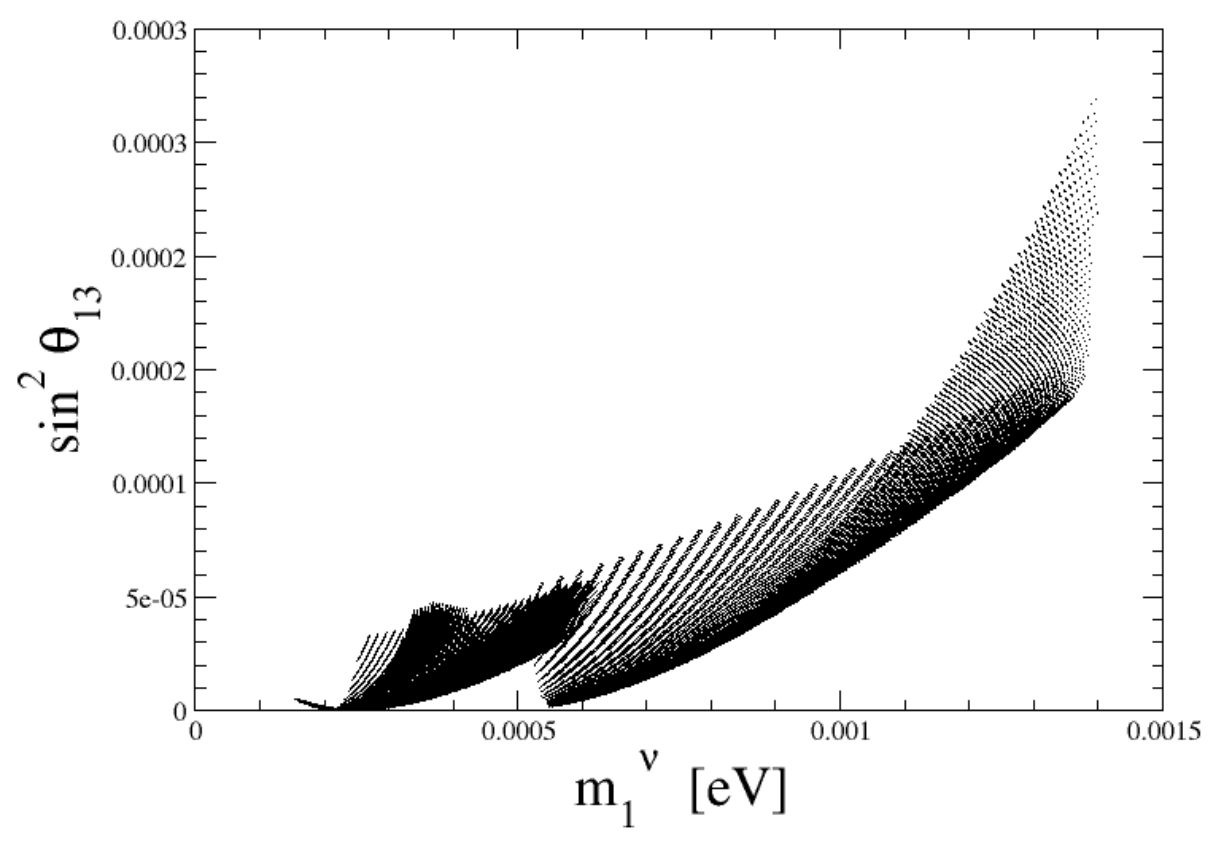

FIG. 1: The predictions of the model in $m_{1}^{\nu}-\sin ^{2} \theta_{13}$ plane.

and [8]

$$
\begin{aligned}
\Delta m_{21} & =(7.45-7.88) \times 10^{-5} \mathrm{eV}^{2}, \Delta m_{31}=(2.29-2.52) \times 10^{-3} \mathrm{eV}^{2}, \\
\sin ^{2} \theta_{12} & =0.288-0.326, \sin ^{2} \theta_{23}=0.44-0.57 .
\end{aligned}
$$

One may think that if we also use $\sin ^{2} \theta_{13}$, we can determine the value of $\epsilon \tau_{0}$ without the result in the quark sector. However, the experimental upper limit of $\sin ^{2} \theta_{13}$ is too mild to constrain the model. Nevertheless, the value of $\sin ^{2} \theta_{13}$ is obtained as a prediction of the model. From the quantities in Eqs. (22) and (23), we get $\alpha_{l} \simeq 82.89^{\circ}$. Hence, our model gives a small $\sin ^{2} \theta_{13}$ as well as FCNCs mediated by the timeon. In Figure 1, we show the allowed values of $m_{1}^{\nu}$ and $\sin ^{2} \theta_{13}$.

\section{B. Leptonic Processes}

As mentioned in the previous subsection, $\alpha_{l} \neq 0$ results in the existence of flavor changing timeon couplings. The branching ratios of the lepton flavor violating (LFV) 
decays due to these coupling are given by [9]

$$
\begin{aligned}
& \operatorname{Br}(\mu \rightarrow e \gamma)=\frac{\alpha_{e m} \tau_{\mu}}{2^{10} \pi^{4}} \frac{m_{\mu}^{3} m_{\tau}^{2}}{M_{T}^{4}}\left(\epsilon F_{\mu \tau} \epsilon F_{e \tau}\right)^{2}\left|\ln \frac{m_{\tau}^{2}}{M_{T}^{2}}+\frac{3}{2}\right|^{2}, \\
& \operatorname{Br}\left(\ell^{-} \rightarrow \ell_{3}^{-} \ell_{2}^{+} \ell_{1}^{-}\right)=\frac{5}{3} \frac{\tau_{\ell}}{2^{11} \pi^{3}} \frac{m_{\ell}^{5}}{M_{T}^{4}}\left(\epsilon F_{\ell_{3} \ell} \epsilon F_{\ell_{2} \ell_{1}}\right)^{2}
\end{aligned}
$$

where $\alpha_{e m}$ is the fine structure constant, $\ell=\mu$ or $\tau, \ell_{i}=e$ or $\mu$, and $\tau_{\ell}$ is the life time of the $\ell$ lepton. From Eqs. (24) and (25), we obtain

$$
\begin{aligned}
& \operatorname{Br}(\mu \rightarrow e \gamma) \simeq 10^{-16}-10^{-21} \\
& \operatorname{Br}\left(\mu^{-} \rightarrow e^{-} e^{+} e^{-}\right) \simeq 10^{-21}-10^{-26}, \\
& \operatorname{Br}\left(\tau^{-} \rightarrow e^{-} e^{+} e^{-}\right) \simeq 3 \times 10^{-21} \\
& \operatorname{Br}\left(\tau^{-} \rightarrow \mu^{-} \mu^{+} \mu^{-}\right) \simeq 10^{-19}-10^{-35}
\end{aligned}
$$

Similarly, we find that the timeon contribution to the muon anomalous magnetic moment is

$$
\begin{aligned}
\Delta a_{\mu} & =\frac{1}{8 \pi^{2}} \frac{m_{\mu}^{2} m_{\tau}^{2}}{M_{T}^{2}}\left(\epsilon F_{\mu \tau}\right)^{2}\left(\ln \frac{m_{\tau}^{2}}{M_{T}^{2}}+\frac{3}{2}\right) \\
& \simeq 10^{-16}-10^{-21} .
\end{aligned}
$$

It is clear that all contributions to the leptonic processes from the timeon are too small to be measured in the near future.

\section{SUMMARY}

We have investigated the timeon model, which is a new kind of spontaneous $C P$ violation mechanism, recently proposed by Friedberg and Lee [3]. However, the original model has a potential problem of FCNCs mediated by the timeon if the timeon mass $M_{T}$ is smaller than $7 \times 10^{5} \mathrm{GeV}$. In order to avoid the problem, we have introduced a small dimensionless parameter to suppress FCNCs and thus allow a smaller $M_{T}$. Due to the constraints from the mass mixing parameters in the $K^{0}-\bar{K}^{0}$ and other neutral meson systems, we have shown that $M_{T} \geq 151 \mathrm{GeV}$. We have also extended the timeon model to the lepton sector. We have found that our simple model predicts a small but non-zero $\sin ^{2} \theta_{13}$ as well as non-zero lepton flavor violating timeon couplings. However, 
all contributions to the LFV decays and muon anomalous magnetic moment due to the timeon are not measurable.

\section{Acknowledgments}

This work is supported in part by the National Science Council of ROC under Grant No: NSC-95-2112-M-007-059-MY3.

[1] N. Cabibbo, Phys. Rev. Lett. 10, 531 (1963); M. Kobayashi and T. Maskawa, Prog. Theor. Phys. 49, 652 (1973).

[2] T. D. Lee, Phys. Rept. 9, 143 (1974).

[3] R. Friedberg and T. D. Lee, arXiv:0809.3633 [hep-ph].

[4] N. Kifune, J. Kubo and A. Lenz, Phys. Rev. D 77, 076010 (2008).

[5] R. Friedberg and T. D. Lee, Annals Phys. 323, 1087 (2008) arXiv:0705.4156 [hep-ph]].

[6] P. F. Harrison, D. H. Perkins and W. G. Scott, Phys. Lett. B 530, 167 (2002); P. F. Harrison and W. G. Scott, Phys. Lett. B 535, 163 (2002).

[7] C. Amsier et al. (Particla Data Group), Phys. Lett. B 667, 1 (2008).

[8] T. Schwetz, M. Tortola and J. W. F. Valle, New J. Phys. 10, 113011 (2008).

[9] J. L. Díaz-Cruz, R. Noriega-Papaqui and A. Rosado, Phys. Rev. D 69, 095002 (2004); Y-F. Zhou, J. Phys. G 30, 783 (2004). 